\title{
Effectiveness of psychotherapy for severe somatoform disorder: meta-analysis
}

Jurrijn A. Koelen, Jan H. Houtveen, Allan Abbass, Patrick Luyten, Elisabeth H. M. Eurelings-Bontekoe, Saskia A. M. Van Broeckhuysen-Kloth, Martina E. F. Bühring and Rinie Geenen

\section{Background}

Patients with severe somatoform disorder (in secondary and tertiary care) typically experience functional impairment associated with physical symptoms and mental distress. Although psychotherapy is the preferred treatment, its effectiveness remains to be demonstrated.

\author{
Aims \\ To examine the effectiveness of psychotherapy for severe \\ somatoform disorder in secondary and tertiary care \\ compared with treatment as usual (TAU) but not waiting-list \\ conditions.
}

\section{Method}

Main inclusion criteria were presence of a somatoform disorder according to established diagnostic criteria and receiving psychotherapy for somatoform disorder in secondary and tertiary care. Both randomised and nonrandomised trials were included. The evaluated outcome domains were physical symptoms, psychological symptoms (depression, anxiety, anger, general symptoms) and functional impairment (health, life satisfaction, interpersonal problems, maladaptive cognitions and behaviour).

\section{Results}

Ten randomised and six non-randomised trials were included, comprising 890 patients receiving psychotherapy and 548 patients receiving TAU. Psychotherapy was more effective than TAU for physical symptoms $(d=0.80 \mathrm{~V}$. $d=0.31, P<0.05)$ and functional impairment $(d=0.45 \mathrm{~V}$. $d=0.15, P<0.01)$, but not for psychological symptoms $(d=0.75 v . d=0.51, P=0.21)$. These effects were maintained at follow-up.

\section{Conclusions}

Overall findings suggest that psychotherapy is effective in severe somatoform disorder. Future randomised controlled studies should examine specific interventions and mechanisms of change.

\section{Declaration of interest}

None.
Somatoform disorders are characterised by persistent physical symptoms that suggest the presence of a medical condition, but are not explained fully by that condition or by the direct effects of substance misuse or mental disorder (DSM-IV). ${ }^{1}$ The prevalence of somatoform disorders is estimated at $6 \%$ in the general population. ${ }^{2}$ Patients with such disorders usually have high functional impairment, ${ }^{3,4}$ are difficult to treat, ${ }^{5,6}$ and show high utilisation of medical care. ${ }^{7}$ Moreover, it typically takes years before they are referred to mental healthcare. ${ }^{6,8,9}$ A strictly somatic approach and unnecessary diagnostic examinations may increase somatising behaviours, ${ }^{10}$ and lead to chronic symptoms and high medical costs: ${ }^{7,11,12}$ these findings emphasise the need for early intervention. ${ }^{13}$ Psychotherapy may be a viable treatment option given the role of behavioural, cognitive and emotional processes in these disorders and their high degree of comorbidity with mental disorders. ${ }^{14-16}$ Some reviews and meta-analyses suggest that psychotherapy may be effective in patients with somatoform disorder. ${ }^{17-19}$ However, these reviews were restricted to psychodynamic psychotherapy only, ${ }^{17}$ or predominantly involved groups with less severe disorder, with functional neurological or conversion disorder generally being excluded. ${ }^{18}$ Hypochondriasis and body dysmorphic disorder were typically included in these reviews, ${ }^{19}$ although it is still a matter of debate whether these conditions should be classified as somatoform disorder. ${ }^{14,20}$ The results of previous reviews cannot always be generalised to patients with strictly defined somatoform disorder in secondary and tertiary care, as these patients are generally more impaired than those seen in primary care. ${ }^{21}$ Finally, previous meta-analyses typically included only randomised trials, often excluding effectiveness studies, ${ }^{22-24}$ whereas the inclusion of both randomised and non-randomised studies allows the meta-analytic comparison of effect sizes between these designs.
The aim of our meta-analysis therefore was to examine the effectiveness of psychotherapy for patients with strictly defined, severe somatoform disorder treated in secondary and tertiary care. To that aim, we compared effect sizes from pre- to post-treatment and from post-treatment to follow-up of psychotherapy and treatment as usual, excluding waiting-list control groups. This study focused on pre- to post-treatment contrasts, and not on between-group contrasts, given the limited number of controlled treatment studies in this context. Given the small number of studies included, moderators of treatment effect were examined only exploratively. We examined methodological quality of the studies, ${ }^{25}$ intervention characteristics (type, modality, frequency and length), ${ }^{26}$ and whether treatment was offered in tertiary (multimodal and integrative) or secondary care settings, ${ }^{18}$ as potential factors influencing treatment effectiveness.

\section{Method}

A multiple-phase search was conducted in March 2010 to retrieve as many studies as possible focusing on the effectiveness of psychological treatments of severe somatoform disorder. 'Severe' disorder was defined as a diagnosis of somatoform disorder according to established criteria and treatment offered in secondary or tertiary care settings. First, studies were retrieved from the Scopus and Web of Science databases with the following search terms: (somatoform ${ }^{\star}$ OR somatisation OR (conversion AND (disorder OR symptoms)) OR (somatoform* AND pain disorder) in the title AND (treatment OR therapy OR intervention OR outcome OR effect ${ }^{*}$ OR efficacy OR evaluation) in the title, abstract or keywords. Second, the reference lists of previous reviews and meta-analyses were hand-searched for additional 
studies. Third, several experts in the field of somatoform disorders were contacted for other studies. Inclusion criteria for the studies were as follows:

(a) a somatoform disorder was present according to DSM-III-R, DSM-IV-TR or ICD-10 diagnostic criteria, with exclusion of hypochondriasis and body dysmorphic disorder, ${ }^{1,27}$ or according to the Somatic Symptom Index, with a lifetime history of eight or more somatic symptoms criterion; ${ }^{28}$

(b) patients received psychotherapy in secondary or tertiary care;

(c) the study reported outcome statistics of validated assessment instruments to allow for effect size calculation;

(d) a prospective design was used;

(e) the publication was in English, Dutch or German;

(f) at least ten patients were included.

Two independent judges applied these selection criteria to the full-text papers that were retrieved, discussed disagreements and decided about definite inclusion. The study selection process is shown in online Fig. DS1. Both randomised and non-randomised (controlled) trials were included.

\section{Data extraction and assessment of study quality}

For each study included, basic within-group (pre- to post-treatment and post-treatment to follow-up) data necessary for effect size calculation were extracted. If necessary, we contacted the authors to obtain additional information. No study had to be excluded because of unavailable data. Effect sizes were calculated for three outcome domains separately. The primary outcome was 'physical symptoms'; secondary outcomes were 'psychological symptoms' (depression, anxiety, anger and general symptoms) and 'functional impairment' (health, life satisfaction, interpersonal problems and maladaptive cognitions and behaviour), as these outcome domains have been commonly used in evaluations of treatments in somatoform disorder. ${ }^{19}$ When a study included multiple variables for the same domain, the means of $z$-transformed variables were used to calculate an average effect size. Self-report measures were included as most studies used self-report instruments only.

Participant information and formal characteristics of the treatments and therapeutic interventions were extracted by a standardised coding scheme. Formal characteristics included setting, duration, frequency, treatment modality, adherence and structure. $^{29}$ To classify therapeutic interventions, categorisation was based on that of De Groot et $a l,{ }^{29}$ and the Comprehensive Psychotherapeutic Interventions Rating Scale (CPIRS) which comprised interventions representative of the following five psychotherapeutic orientations: experiential, psychodynamic, directive-behavioural, cognitive and systemic. ${ }^{26}$ We adapted this scale for the treatment of somatoform disorder by adding 'body-directed interventions'. The intervention was rated as 'important' (explicitly mentioned as core component), 'possibly important' (implicit as core component), 'possibly not important' (uncertain whether included or not) and 'not important' (not mentioned and definitely not a core component).

Studies included were rated by two independent judges on methodological quality with the revised Psychotherapy Quality Rating Scale (PQRS). ${ }^{30}$ This scale comprised 25 items covering six domains: description of patients (four items); definition and delivery of treatment (five items); outcome measures (five items); data analysis (five items); treatment assignment (three items); and overall quality (three items). The last item was scored on a sevenpoint scale and the other items on a three-point scale. Some minor adaptations to the scale were made based on the Strengthening the
Reporting of Observational Studies in Epidemiology (STROBE) statement recommendations for cohort studies to render it suitable for quasi-experimental and uncontrolled studies. ${ }^{31}$ Items were added to yield information about repeated measurements, to evaluate sources of bias in uncontrolled trials as a consequence of recruitment procedures and non-random allocation to treatment groups and their impact on study generalisability, and concerning the matching of groups on clinical and demographic variables.

Two independent judges coded all studies to establish interrater reliability (across 14 studies). The one-way random intraclass correlation (ICC) single measures was 0.91 and the ICC average measures was 0.95 , which is excellent. ${ }^{32}$

\section{Statistical analysis}

\section{Effect sizes}

Standardised mean pre- to post-psychotherapy changes were evaluated. Effect sizes (Cohen's $d$ ) were calculated by subtracting post-treatment scores from pre-treatment scores and dividing these by the pooled standard deviations. To calculate the pooled s.d. the test-retest reliability for the most common instrument in that domain was taken into account. Follow-up effect sizes were calculated by subtracting follow-up scores from post-treatment scores and dividing by the pooled s.d. to determine the sustainability of effects. We selected the longest time frame between treatment termination and follow-up. Effect sizes of $0.20,0.50$ and 0.80 are considered small, medium and large respectively; effect sizes below 0.20 are considered very small. ${ }^{33}$ Effect sizes were weighted by their inverse variance. ${ }^{34}$ This procedure gives more weight to larger studies (with smaller sampling error), thus increasing the reliability of the effect estimates.

Within the same study, effect sizes were calculated separately for one or more treatments and for the treatment as usual (TAU) control condition. To be regarded as TAU, treatments had to be specified by the researchers as such or as (standard or enhanced) medical care, psychiatric advice to the general practitioner or TAU with a small component added (e.g. relaxation or gymnastics). Waiting-list control groups were not considered to be TAU. When the alleged comparison condition clearly included psychotherapy as a main component, the condition was categorised as psychotherapy.

\section{Pooled effect sizes across studies}

Pooled effect sizes were calculated with the Comprehensive Metaanalysis (CMA) software version 2.0 on Windows (Biostat, Englewood, New Jersey, USA). A random effects model was used to compute weighted mean effect sizes, because we expected true population effect sizes to vary across studies owing to differences in sample, methodology and treatment. The random effects model results in more conservative results and broader 95\% confidence intervals than the fixed effects model. ${ }^{35}$ Heterogeneity of effect sizes within and between subsamples was calculated using the $Q$ statistic. ${ }^{35}$ Pooled effect sizes were also recalculated with outliers removed when the individual effect sizes did not overlap with the confidence interval.

\section{Moderator analysis}

To examine potential moderators of treatment outcome, associations between effect sizes and the following dichotomous (yes/no) variables were calculated with CMA: group psychotherapy (with or without additional individual psychotherapy); treatment setting (out-patient $v$. in-patient); high or low treatment 
frequency (more than two sessions per week); treatment manual available; adherence check; supervision of therapists; additional pharmacotherapy allowed; randomised or non-randomised trial; intention-to-treat analysis; multicomponent (treatment components besides psychotherapy); cognitive interventions; behavioural interventions; psychodynamic interventions; affective interventions; interpersonal interventions; experiential interventions; and body-directed interventions. For the purpose of moderator analyses, studies were divided into subgroups. For each subgroup the pooled mean effect size was calculated, and differences in effect sizes between the subgroups (with a minimum of three studies) were examined for statistical significance using the $Q$ statistic. Meta-regression analyses were conducted to explore whether gender, age, prevalence of diagnosis of somatisation disorder, methodological quality of the study (according to the Kocsis et al checklist) and length of treatment predicted effect sizes. ${ }^{30}$ Moderator analyses were performed only for pre-post contrasts and not for follow-up, because the samples were too small to yield meaningful effects.

\section{Publication bias}

Meta-analyses typically contain an overrepresentation of published studies that are biased towards statistically significant findings. ${ }^{36}$ We tested potential publication bias by means of the iterative non-parametric trim and fill procedure as implemented in CMA. ${ }^{37}$ This procedure controls for the association between individual effect sizes and their sample sizes (sampling error) by inspecting funnel plots: publication bias is present when the effect sizes of small studies with larger sampling variation than large studies are represented asymmetrically within and around the funnel. The Duval \& Tweedie procedure provides a correction of the effect size after publication bias has been taken into account by trimming away studies suggesting asymmetry. ${ }^{37}$ We used the random effects model. Publication bias was also evaluated with Orwin's fail-safe $N$ statistic, which expresses how many studies would bring the combined effect to a specified level other than zero. $^{38}$ The criterion was set to $d=0.10$.

\section{Results}

Sixteen studies were included in the meta-analysis (online Fig. DS1). ${ }^{4,6-10,12,39-47}$ The main characteristics are summarised in online Table DS1. Fourteen studies focused exclusively on somatoform disorder; two studies included patients with a range of mental disorders but were eligible because statistics for the somatoform disorder subgroup were reported separately. ${ }^{39,40}$ All studies but one were published after $2000 .{ }^{41}$ Ten studies were conducted in Germany, ${ }^{4,6,7,9,12,39,40,45-47}$ two in the USA, ${ }^{8,10}$ two in The Netherlands, ${ }^{43,44}$ one in Turkey, ${ }^{42}$ and one in China. ${ }^{41}$

\section{Characteristics of treatment}

In the included studies the intention-to-treat sample comprised 936 participants in the psychotherapy conditions of whom 854 (91\%) completed treatment and 890 (95\%) were eventually included in analyses. In the TAU conditions the intention-to-treat sample comprised 568 patients of whom 519 (91\%) patients actually completed their treatment and 548 (96\%) were included in analyses. The median duration of psychotherapy was 9.2 weeks (range 2-25) for all but one study which provided more than 250 sessions over on average 178 weeks. ${ }^{46}$ In the TAU groups, patients received treatment for a median of 9 weeks (range 5.5-12). Other relevant information regarding the study characteristics and therapeutic interventions is reported in online Tables DS1 and DS2.

\section{Characteristics of participants}

The mean age of the treated patients was 39.7 years (s.d. = 8.1). All studies included adults except for one study in which the age range was $16-30$ years. ${ }^{42}$ The percentage of women across the studies was $67 \%(n=950)$. The mean duration of physical symptoms (reported in ten studies) was 3135 days $($ s.d. $=2564$ ), i.e. about 8.6 years. Eleven studies provided information about comorbid diagnoses: the most common were mood and anxiety disorders. Two studies excluded one patient with depression. ${ }^{43,44}$

\section{Methodological characteristics}

The mean methodological quality across 16 studies according to the revised PQRS was 21.0 (range 10-36), indicating on average a moderately poor study quality (ranging from very poor to very good). The mean methodological quality of the ten randomised controlled trials (RCTs) was rated 25.1 (range 17-36) v. 15.0 (range 12-21) for the six non-RCTs. Twelve studies used manualised treatments or provided sufficient information about the treatment to allow replication. Four of these studies conducted a treatment integrity check. ${ }^{6,8,43,44}$ Six studies with manualised treatments provided regular supervision of the therapists. ${ }^{6,8,40,43,44,47}$ Other treatments such as pharmacotherapy were stopped during the treatment period in only two studies. ${ }^{41,42}$ One study provided paradoxical therapy, which was compared with diazepam. ${ }^{42}$ One study examined the effect of acupuncture in addition to cognitive-behavioural therapy (CBT). ${ }^{41}$ In six studies no explicit mention was made of pharmacotherapy. In one study, outcome assessments were made by a psychiatrist who was masked to the treatment conditions of the patients throughout the study. ${ }^{42}$ Three studies used partial masking (only on some outcome instruments), ${ }^{7,43,44}$ and one study contained no information as to whether assessments were conducted masked to treatment condition. ${ }^{41}$ Most studies conducted intention-to-treat analyses including patients who withdrew from treatment in the analyses. Ten studies carried out follow-up assessments; the mean follow-up period across these studies was 10.7 months (range 5-22) after treatment termination.

\section{Pre-post pooled effect sizes}

Figures 1-3 summarise the pooled weighted pre-post effect sizes, test statistics and heterogeneity indices for psychotherapy and TAU for three outcome domains separately.

\section{Physical symptoms}

The pooled effect size of physical symptoms for psychotherapy across nine studies was large $(d=0.80)$. Treatment as usual $(4$ studies) yielded a small pooled effect size $(d=0.31)$. Heterogeneity based on the $Q$ statistic was high and significant in both conditions. Analyses with outliers removed yielded similar results. Comparison of pooled effect sizes yielded a superior outcome for patients receiving psychotherapy ( 9 studies) compared with TAU (4 studies): $Q=5.4, P<0.05$. Moderator analyses for this domain showed lower effect sizes for studies with supervision of therapists (3 studies) v. studies without (6 studies) (online Fig. DS2). Metaregression showed a better outcome for younger patients (slope $-0.036, P<0.01$ ), women (slope $0.007, P<0.03$ ), patients with somatisation disorder (slope 0.004, $P<0.01$ ) and studies with lower methodological quality (slope $-0.016, P<0.01$ ). Treatment length was not a moderator (slope 0.001 , not significant).

\section{Psychological symptoms}

The pooled effect size of psychological symptoms for psychotherapy across 14 studies was moderate to large $(d=0.75)$. Treatment as 
Study

Allen et al $(2001)^{10}$

Allen et al (2006)

Bleichhardt et al (2004) $45, a$

Bleichhardt et al (2004) $)^{45, a}$

Hiller et al (2003) ${ }^{12}$

Leichsenring et al (2008) ${ }^{39}$

Nickel et al (2006) ${ }^{46}$

Sattel et al (2012)

Zaby et al $(2008)^{47}$

Total

Test for heterogeneity: $Q=367.5^{\star *}$

Study

Allen et al (2006) ${ }^{8}$

Nickel et al (2006) $)^{46}$

Sattel et al (2012) ${ }^{6}$

Zaby et al (2008) ${ }^{47}$

Total

Test for heterogeneity: $Q=58.9 * *$
Psychotherapy

$d(95 \% \mathrm{Cl})$ $0.71(0.40-1.02)$ $0.74(0.59-0.89)$ $0.80(0.69-0.91)$ $0.68(0.56-0.80)$ $0.45(0.38-0.52)$ $0.91(0.62-1.19)$ $2.36(2.14-2.57)$ $0.27(0.18-0.35)$ $0.31(0.12-0.50)$ $0.80(0.50-1.09)$

d $(95 \% \mathrm{Cl})$

$0.15(0.02-0.29)$ $0.71(0.59-0.83)$

$0.16(0.07-0.24)$ $0.23(0.03-0.42)$ $0.31(0.03-0.60)$

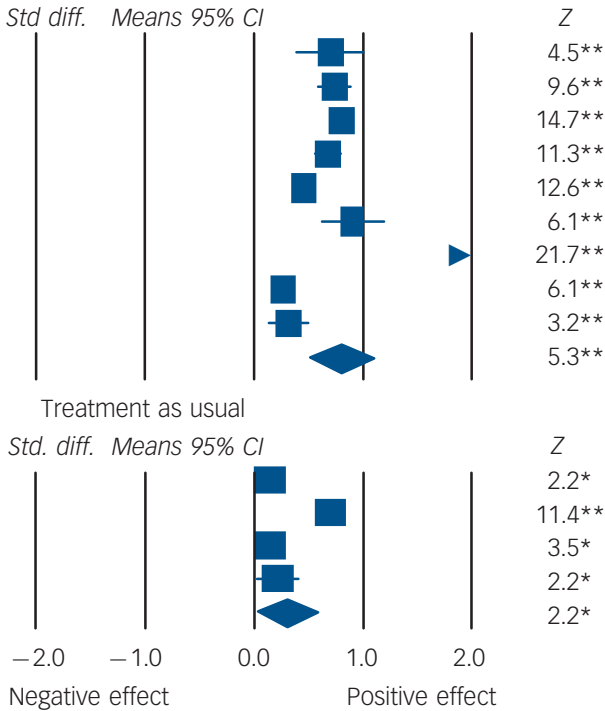

$4.7 * *$

$1.3^{* \star}$

$2.6^{* *}$

$6.1^{* *}$

$61 * *$

$2 * *$

$.3^{\star \star}$

$1.4^{\star *}$

$3.5^{*}$

$2^{*}$
Negative effect Positive effect

Fig. 1 Physical symptoms: pre- to post-treatment effects.

a. The study by Bleichhardt et al is listed twice because both conditions were analysed as active psychotherapeutic treatments. ${ }^{*} P<0.05, * * P<0.01$.

Study

Ataoglu et al (2003) $)^{42}$

Bleichhardt et al (2004) $)^{45, a}$

Bleichhardt et al (2004) 45 , a

Dongfen \& Shizong (1999) ${ }^{41, a}$

Dongfen \& Shizong (1999) $)^{41, a}$

Hiller et al (2003) ${ }^{12}$

Junkert-Tress et al (2001) ${ }^{40}$

Leichsenring et al (2008) ${ }^{39}$

Moene et al (2002) ${ }^{43}$

Moene et al $(2003)^{44}$

Nickel et al (2006) ${ }^{46}$

Sattel et al (2012)

Tschuschke et al (2007)

Zaby et al (2008) $)^{47}$

Total

Test for heterogeneity: $Q=89.7^{* *}$

Study

Ataoglu et al (2003) ${ }^{42}$

Moene et al (2002) ${ }^{43}$

Nickel et al (2006) ${ }^{46}$

Sattel et al (2012) ${ }^{6}$

Zaby et al $(2008)^{47}$

Total

Test for heterogeneity: $Q=25.8^{\star *}$ d $(95 \% \mathrm{Cl})$

$2.53(1.70-3.36)$

$0.64(0.46-0.83)$

$0.67(0.46-0.88)$

$1.22(0.95-1.48)$

$0.72(0.49-0.95)$

$0.84(0.70-0.98)$

$0.80(0.42-1.18)$

$1.25(0.67-1.83)$

$0.10(-0.22-0.42)$

$0.20(-0.15-0.56)$

$1.18(0.92-1.43)$

$0.44(0.29-0.60)$

$0.50(0.27-0.74)$

$0.51(0.15-0.87)$

$0.75(0.57-0.92)$

$d(95 \% \mathrm{Cl})$

1.86 (1.19-2.53)

$0.47(0.11-0.83)$

$0.47(0.26-0.68)$

$0.22(0.06-0.37)$

$0.12(-0.24-0.47)$

$0.51(0.19-0.83)$
Psychotherapy

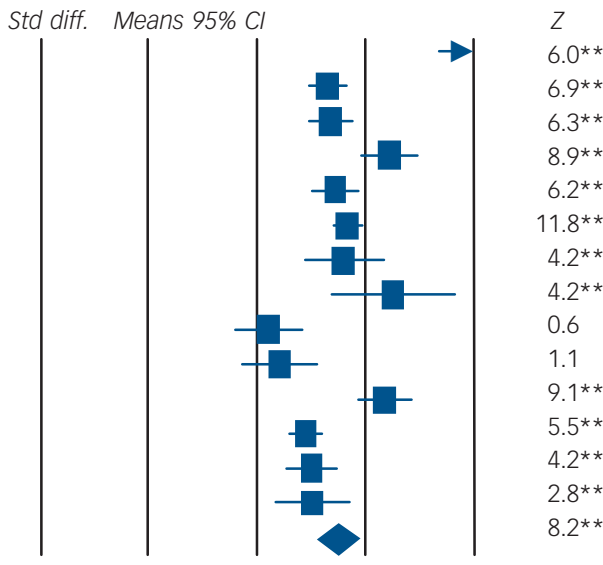

Treatment as usual

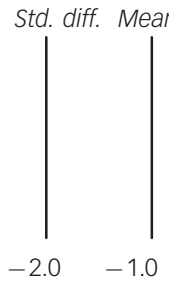

Negative effect 


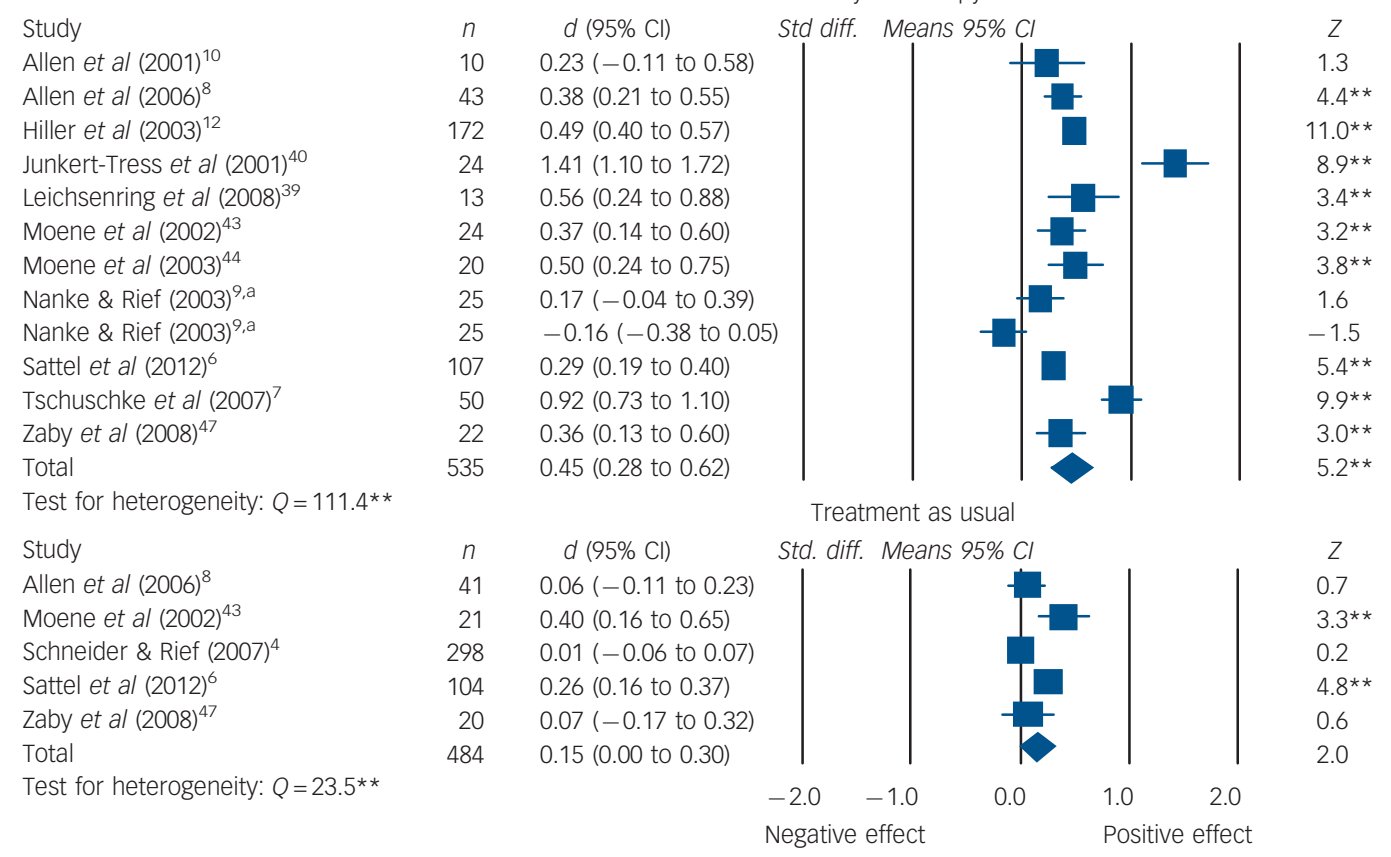

Fig. 3 Functional impairment: pre- to post-treatment effects.

Heterogeneity was high and significant in both conditions. Analyses with outliers removed yielded similar results. Comparison of the pooled effect sizes indicated a better outcome for patients receiving psychotherapy (12 studies) than those receiving TAU (5 studies): $Q=6.8, P<0.01$. Moderator analyses in this outcome domain showed a better outcome for studies without cognitive and behavioural interventions, studies without body-directed therapeutic interventions and non-RCTs (online Fig. DS2). When compared head to head, psychodynamic interventions were more effective than cognitive interventions: $d=0.79$ (4 studies) $v$. $d=0.27$ (7 studies), $P<0.05$. Meta-regression yielded better results for younger patients (slope $-0.027, P<0.01$ ), men (slope $-0.012, P<0.01$ ), patients with somatisation disorder (slope $-0.004, P<0.01$ ), studies with lower methodological quality (slope $-0.016, P<0.01$ ) and longer treatments (slope $0.002, P<0.05$ ).

\section{Publication bias}

Some evidence of publication bias was found for physical symptoms and functional impairment, but not for psychological symptoms. The pooled pre-post treatment effect size for physical symptoms remained large after adjustment for publication bias $(d=1.03$, 95\% CI $0.65-$ 1.41); three studies were trimmed. Orwin's fail-safe $N$ showed that 44 missing studies with an average effect size of zero would need to be published to render the significant results clinically irrelevant (mean effect size $d=0.10$ ). The pre-post treatment effect size for functional impairment remained moderate after correction for publication bias $(d=0.57,95 \%$ CI $0.39-0.76)$; three studies were trimmed. Orwin's fail-safe $N$ indicated that 40 missing studies with a zero effect size would need to be published to bring the significant results to the specified irrelevant level of $d=0.10$. In sum, although there was some evidence of publication bias, the results essentially remained similar when adjusted for this bias.

\section{Post-treatment to follow-up pooled effect sizes}

Table 1 summarises the effect sizes and test statistics from posttreatment to follow-up for psychotherapy and TAU. For physical and psychological symptoms there was no significant change between treatment termination and follow-up. On measures of functional impairment patients who had received psychotherapy rather than TAU continued to improve. There was no posttherapy to follow-up difference between psychotherapy and TAU on any outcome domain (physical symptoms $Q=0.0, P=0.93$; psychological symptoms $Q=0.0, P=0.83$; functional impairment $Q=0.7, P=0.39$ ). These findings indicate that after treatment the psychotherapy and TAU patient groups changed in a similar fashion. Our findings further imply that all gains immediately after treatment were sustained at follow-up, for both psychotherapy and TAU, with continuing improvement in the domains of health and interpersonal functioning for psychotherapy.

\section{Discussion}

This meta-analysis examined the effectiveness of psychotherapy for severe somatoform disorder in secondary and tertiary care. Within specific limitations, such as heterogeneity of studies and limited methodological quality, overall findings suggest that psychotherapy is effective in severe somatoform disorder. Pre- to post-therapy improvements were large for physical symptoms, medium to large for psychological symptoms and small to medium for functional impairment. For TAU, effect sizes were small for physical symptoms, medium for psychological symptoms and very small for functional impairment. The differences in effect size between psychotherapy and TAU were significant for physical symptoms and functional impairment. These findings suggest that psychotherapy is more effective than TAU in severe somatoform disorder, particularly in terms of reduction of physical symptoms and functional impairment. This meta-analysis extends previous research in at least three ways. It is the first to indicate that psychotherapy is more effective than TAU for strictly defined, severe and chronic somatoform disorder, which is considered a debilitating and difficult-to-treat disorder. $^{6,12}$ Second, since the previous meta-analysis new empirical data have been published that are included in our 


\begin{tabular}{l} 
Table 1 Post-treatment to follow-up: weighted effect sizes and relevant test statistics \\
\cline { 2 - 5 }
\end{tabular}

meta-analysis. Finally, the inclusion criteria were different from those used in previous reviews and meta-analyses - for example, functional neurological or conversion disorder was not included in earlier meta-analyses.

The effects found for psychotherapy were maintained nearly a year after treatment. For functional impairment the effect during treatment was smaller than for the symptom domains, yet results suggested a small but significant continuing decrease in functional impairment during follow-up. This finding is congruent with studies suggesting that improvements in patients' general functioning may take considerable time given the chronic nature of this disorder and the severity of functional impairment. ${ }^{48,49}$ Dose-effect studies indicate that significant improvements in characterological, interpersonal and social functioning generally require at least 6 months of psychotherapy. ${ }^{50}$ Our findings do not confirm this suggestion for functional impairment, but the continued improvement at the long-term follow-up in this domain may reflect that improvement in symptoms often precedes improvement in general functioning. ${ }^{50,51}$ Given the chronic nature of somatoform disorders and the high levels of associated functional impairment, it is remarkable that extant research has mainly focused on studies of the effectiveness of brief psychotherapies. It is relevant here that within these brief treatments, longer treatment duration was associated with larger effects in terms of general functioning.

Effect sizes for psychotherapy for somatoform disorder were generally lower than those typically found for mental disorders. For instance, one meta-analysis reported higher pre- to post-treatment effect sizes of short-term psychodynamic therapies for depression $(d=1.30-1.87),{ }^{52}$ compared with the effect sizes for somatoform disorder in this meta-analysis $(d=0.45-0.80)$. There are several possible reasons for this. First, treatments for depression have been more systematically developed and examined compared with those for somatoform disorder. Second, clinically relevant changes may be easier to achieve in a syndrome with an episodic nature such as depression than in a more stable syndrome such as somatoform disorder. Third, patients with somatoform disorder, who often are subjected to redundant medical examinations over many years, may show much longer delay in seeking psychotherapy than patients with depression. ${ }^{13}$

\section{Moderators}

A secondary aim of our meta-analysis was to examine moderators of treatment effects. However, moderator analyses reported here should be interpreted with considerable caution. Because they are intercorrelated owing to the relatively small number of studies, the moderators in this analysis may be statistically associated with outcomes, but the underlying mechanism may be very different and not (fully) captured by the moderator. Within these limitations, findings indicated that interpersonal interventions were less effective in reducing psychological symptoms. Furthermore, cognitive and behavioural therapies and therapies that mainly focus on the body may not be effective in reducing functional impairment. Some studies have emphasised the importance of emotional awareness, ${ }^{6,17,53}$ emotion exposure and expression when dealing with patients with somatoform disorder, ${ }^{54,55}$ as well as their interpersonal difficulties; ${ }^{56,57}$ all of these are core features of psychodynamic therapy. ${ }^{58,59}$ Concordant with this notion, post hoc analysis indicated that psychodynamic interventions were more effective in improving functioning than cognitive interventions, although not in improving symptoms. Future research directly comparing these interventions is needed to replicate these findings before any substantial conclusions can be drawn. It is relevant that our meta-analysis indicates that other forms of psychotherapy besides CBT are potentially effective for severe somatoform disorder. This finding suggests that different mechanisms, rather than a single mechanism, may underlie therapeutic change in patients with severe somatoform disorder. ${ }^{49}$

Nearly half of the studies were conducted in clinical settings involving intensive, multimodal treatments; however, these treatments were not superior to out-patient treatment. Hence, high-frequency and integrative multicomponent treatments may not be more effective than less intensive - and probably less expensive - out-patient treatments. Yet, it is also possible that pre-treatment variables associated with differential referral to out-patient and in-patient care are responsible for these findings. Moreover, we found no difference in the effectiveness of group $v$. individual therapy; this is in contrast to results from a previous meta-analysis that included also patients with less severe somatoform disorder, ${ }^{18}$ underscoring the need for a separate meta-analysis focusing on patients with severe disorder only. Generally, elderly patients benefited less from psychotherapy, which indicates that some elements of the treatment may need to be adapted to the patient's age. Women benefited more from treatment than men in terms of physical symptoms, but not psychological symptoms or functional impairment. Because women tend to have more physical symptoms than men, especially in clinical samples, ${ }^{60}$ there may simply be more room for symptom improvement in female patients. A similar argument might apply to more symptomatic somatoform disorder, as physical and psychological symptoms improved more in studies with a higher percentage of patients diagnosed with somatisation disorder. 
Congruent with another meta-analysis, ${ }^{25}$ smaller effect sizes were found in studies of higher methodological quality. In some outcome domains stricter control over the delivery of treatment (e.g. manual or adherence check) or the study design (RCT $v$. non-RCT) yielded lower effect sizes. Studies of low methodological quality are likely to include fewer experimentally controlled factors that might subsequently result in higher effectiveness. ${ }^{25}$ Second, higher methodological quality might actually lead to therapists relying more rigidly on the treatment manual, which might hamper the flexibility that is needed in applying any treatment protocol. ${ }^{49}$ This finding emphasises the importance of including a range of study designs in the examination of the effectiveness of treatments, especially in the early stages of treatment development. ${ }^{22}$

\section{Limitations}

The results of this meta-analysis should be interpreted within the context of its methodological limitations. First, the small number of studies limited the ability to draw definite conclusions. Second, heterogeneity of studies and outcome measures may have influenced results, although we used conservative confidence intervals for the estimation of effect sizes. Third, publication bias was present, although did not appear to have significantly influenced the results. Fourth, study quality was moderately poor on average. Fifth, most outcome assessments relied only on patients' self-reports. Sixth, the use of concomitant medications was not reported in each study. Seventh, a meta-analysis of between-group contrasts was not feasible, because three of the retrieved effectiveness studies were non-inferiority trials comparing two forms of CBT and seven studies had no control treatment, thus hampering between-group comparisons. Eighth, as all studies involved patients with severe somatoform disorder, results from the meta-analysis do not generalise to syndromes labelled 'functional somatic syndrome', 'medically unexplained physical symptoms' or 'abridged somatisation disorder', and chronic diseases with multiple physical symptoms. Finally, in light of recent changes in diagnostic classification, it remains uncertain how the findings in this group of somatoform disorders will translate to the revised diagnostic system in DSM-5. ${ }^{14,61,62}$

\section{Future research}

Future studies should aim to examine cost-effectiveness of various forms of psychotherapy given the excessively high medical costs and loss of productivity associated with somatoform disorder. Given the limited quality of the existing body of studies, future research should include more randomised controlled studies of higher quality. This would allow comparisons between potentially effective psychotherapies for (severe) somatoform disorder or 'somatic symptom syndromes, ${ }^{14}$ and more focused hypothesis testing of specific interventions and potential mechanisms of change.

Jurrijn A. Koelen, MSc, Altrecht Psychosomatic Medicine, Zeist, The Netherlands, and Department of Psychology, University of Leuven, Leuven, Belgium; Jan $\mathbf{H}$. Houtveen, PhD, Department of Clinical and Health Psychology, Utrecht University, Utrecht, The Netherlands; Allan Abbass, MD, PhD, Departments of Psychiatry and Psychology, Dalhousie University, Halifax, Canada; Patrick Luyten, PhD, Faculty of Psychology and Educational Sciences, University of Leuven, Leuven, Belgium, and Psychology and Educational Sciences, University of Leuven, Leuven, Belgium, and Research Department of Clinical, Educational and Health Psychology, University
College London, UK; Elisabeth H. M. Eurelings-Bontekoe, PhD, Department of Clinical and Health Psychology, Leiden University, Leiden; Saskia

A. M. Van Broeckhuysen-Kloth, PhD, Martina E. F. Bühring, MD, PhD, Altrecht Psychosomatic Medicine, Zeist; Rinie Geenen, PhD, Department of Clinical and Health Psychology, Utrecht University, Utrecht, The Netherlands

Correspondence: J. A. Koelen, University of Leuven, Tiensestraat 102, 3000 Leuven, Belgium. Email: jurkoel@gmail.com

First received 2 Oct 2012, final revision 18 Jun 2013, accepted 20 Jun 2013

\section{Funding}

The meta-analysis was funded in part by a voucher of SenterNovem grant X090109.

\section{Acknowledgements}

The authors thank F. Stuke for her contributions in rating the methodological quality of the individual studies, E. Driessen for her helpful suggestions concerning the software and the analyses, H. Sattel and F. Leichsenring for providing additional data, and N. Thoma for his advice concerning the Psychotherapy Quality Rating Scale.

\section{References}

1 American Psychiatric Association. Diagnostic and Statistical Manual of Mental Disorders (4th edn) (DSM-IV). APA, 1994.

2 Wittchen HU, Jacobi F, Rehm J, Gustavsson A, Svensson M, Jonsson B, et al. The size and burden of mental disorders and other disorders of the brain in Europe 2010. Eur Neuropsychopharm 2011; 21: 655-79.

3 De Waal MWM, Arnold IA, Eekhof JAH, Van Hemert AM. Somatoform disorders in general practice. Prevalence, functional impairment and comorbidity with anxiety and depressive disorders. Br J Psychiatry 2004; 184: $470-6$.

4 Schneider J, Rief W. Selbstwirksamkeitserwartungen und Therapieerfolge bei Patienten mit anhaltender somatoformer Schmerzstörung [Self-efficacy and therapeutic success in patients with chronic somatoform pain disorder]. Z KI PSych Psychoth 2007; 36: 46-56.

5 Hahn SR. Physical symptoms and physician-experienced difficulty in the physician-patient relationship. Ann Intern Med 2001; 134: 897-904.

6 Sattel H, Lahmann C, Gündel H, Guthrie E, Kruse J, Noll-Hussong M, et al. Brief psychodynamic interpersonal psychotherapy for patients with multisomatoform disorder: randomised controlled trial. Br J Psychiatry 2012; 200: 60-7.

7 Tschuschke V, Weber R, Horn E, Kiencke P, Tress W. Ambulante psychodynamische Kurzgruppenpsychotherapie bei Patienten mit somatoformen Störungen [Out-patient short-term psychodynamic group psychotherapy in patients with somatoform disorders]. Z Psychiatr Psych PS 2007; 55: 87-95.

8 Allen LA, Woolfolk RL, Escobar Jl, Gara MA, Hamer RM. Cognitive-behavioral therapy for somatization disorder. A randomized controlled trial. Arch Intern Med 2006; 166: 1512-8.

9 Nanke A, Rief W. Biofeedback-based interventions in somatoform disorders: a randomized controlled trial. Acta Neuropsychiatr 2003; 15: 249-56.

10 Allen LA, Woolfolk RL, Lehrer PM, Gara MA, Escobar Jl. Cognitive behavior therapy for somatization disorder: a preliminary investigation. $J$ Behav Ther Exp Psychiatry 2001; 32: 53-62.

11 Barsky AJ, Orav EJ, Bates DW. Somatization increases medical utilization and costs independent of psychiatric and medical comorbidity. Arch Gen Psychiatry 2005; 62: 903-10.

12 Hiller W, Fichter MM, Rief W. A controlled treatment study of somatoform disorders including analysis of healthcare utilization and cost-effectiveness. J Psychosom Res 2003; 54: 369-80.

13 Bouton $C$, Roche G, Roquelaure $Y$, Legrand $E$, Penneau-Fontbonne D, Dubus $\mathrm{V}$. Management of low back pain in primary care prior to multidisciplinary functional restoration: a retrospective study of 72 patients. Ann Readapt Med Phys 2008; 51: 650-62.

14 American Psychiatric Association. DSM-5 Development. Somatoform Disorders. APA, 2012.

15 Bass C, Murphy M. Somatoform and personality disorders: syndromal comorbidity and overlapping developmental pathways. J Psychosom Res 1995; 39: 403-27.

16 Sar V, Akyuz G, Kundakci T, Kiziltan E, Dogan O. Childhood trauma, dissociation, and psychiatric comorbidity in patients with conversion disorder. Am J Psychiatry 2004; 161: 2271-6.

17 Abbass A, Kisely S, Kroenke K. Short-term psychodynamic psychotherapy for somatic disorders. Psychother Psychosom 2009; 78: 265-74.

18 Kleinstauber M, Witthoft M, Hiller W. Efficacy of short-term psychotherapy for multiple medically unexplained physical symptoms: a meta-analysis. Clin Psychol Rev 2011; 31: 146-60.

19 Kroenke K. Efficacy of treatment for somatoform disorders: a review of randomized controlled trials. Psychosom Med 2007; 69: 881-8.

20 Mayou R, Kirmayer $\amalg$, Simon G, Kroenke K, Sharpe M. Somatoform disorders: time for a new approach in DSM-V. Am J Psychiatry 2005; 162: 847-55. 
21 Van der Feltz-Cornelis C, Van Oppen P, Ader HJ, Van Dyck R. Randomized controlled trial of a collaborative care model with psychiatric consultation for persistent medically unexplained symptoms in general practice. Psychother Psychosom 2006; 75: 282-9.

22 Morrison $\mathrm{KH}$, Bradley R, westen D. The external validity of controlled clinical trials of psychotherapy for depression and anxiety: a naturalistic study. Psychol Psychother 2003; 76: 109-32.

23 Leichsenring F. Randomized controlled versus naturalistic studies: a new research agenda. Bull Menninger Clin 2004; 68: 137-51.

24 Westen D, Novotny CM, Thompson-Brenner $\mathrm{H}$. The empirical status of empirically supported psychotherapies: assumptions, findings, and reporting in controlled clinical trials. Psychol Bull 2004; 130: 631-63.

25 Thoma NC, McKay D, Gerber AJ, Milrod BL, Edwards AR, Kocsis JH. A qualitybased review of randomized controlled trials of cognitive-behavioural therapy for depression: an assessment and metaregression. Am J Psychiatry 2012; 169: 22-30.

26 Trijsburg RW, Frederiks GCFJ, Gorlee M, Klouwer E, den Hollander AM, Duivenvoorden $\mathrm{HJ}$. Development of the comprehensive psychotherapeutic interventions rating scale (CPIRS). Psychother Res 2002; 12: 287-317.

27 World Health Organization. The ICD-10 Classification of Mental and Behavioural Disorders. WHO, 1992.

28 Hiller W, Rief W, Fichter MM. Further evidence for a broader concept of somatization disorder using the Somatic Symptom Index (SSI). Psychosomatics 1995; 36: 285-94.

29 De Groot ER, Verheul R, Trijsburg RW. An integrative perspective on psychotherapeutic treatments for borderline personality disorder. J Personal Disord 2008; 22: 332-52.

30 Kocsis JH, Gerber AJ, Milrod B, Roose SP, Barber J, Thase ME, et al. A new scale for assessing the quality of randomized clinical trials of psychotherapy. Compr Psychiatry 2010; 51: 319-24.

31 Von Elm E, Altman DG, Egger M, Pocock SJ, Gotzsche PC, Vandenbroucke JP, et al. The Strengthening the Reporting of Observational Studies in Epidemiology (STROBE) statement: guidelines for reporting observational studies. Lancet 2007; 370: 1453-7.

32 Shrout $\mathrm{PE}$, Fleiss JL. Intraclass correlations: uses in assessing rater reliability. Psychol Bull 1979; 86: 420-8

33 Cohen J. Statistical Power Analysis for the Behavioural Sciences. Academic Press, 1977.

34 Lipsey MW, Wilson DB. Practical Meta-Analysis. Sage, 2001.

35 Borenstein M, Hedges LV, Higgins JPT, Rothstein HR. Introduction to Meta-Analysis. Wiley, 2009.

36 Higgins JP, Thompson SG, Deeks JJ, Altman DG. Measuring inconsistency in meta-analyses. BMJ 2003; 327: 557-60.

37 Duval S, Tweedie R. Trim and fill: a simple funnel-plot-based method of testing and adjusting for publication bias in meta-analysis. Biometrics 2000; 56: $455-63$.

38 Borenstein M. Software for publication bias. In Publication Bias in MetaAnalysis - Prevention, Assessment and Adjustments (eds HR Rothstein, AJ Sutton, M Borenstein): 193-220. Wiley, 2005.

39 Leichsenring F, Kreische R, Biskup J, Staats H, Rudolf G, Jakobsen T. Die Göttinger Psychotherapiestudie. Ergebnisse analytischer Langzeitpsychotherapie bei depressiven Störungen, Angststörungen, Zwangsstörungen, somatoformen Störungen, und Personlichkeitsstörungen [Outcome of long-term psychoanalytic psychotherapy in depression, anxiety disorders, obsessive-compulsive disorders, somatoform disorders and personality disorders]. Forum Psychoanal 2008; 24: 193-204.

40 Junkert-Tress B, Schnierda U, Hartkamp N, Schmitz N, Tress W. Effects of short-term dynamic psychotherapy for neurotic, somatoform, and personality disorders: a prospective 1-year follow-up study. Psychother Res 2001; 11: 187-200.

41 Dongfen $\mathrm{H}$, Shizong $\mathrm{S}$. Therapeutic effects of acupuncture combined with psychotherapy for patients with somatoform disorders. In Somatoform Disorders: A Worldwide Perspective (eds Y Ono, A Janca, M Asai, N Sartorius): 253-6. Springer, 1999.
42 Ataoglu A, Ozcetin A, Icmeli C, Ozbulut O. Paradoxical therapy in conversion reaction. J Korean Med Sci 2003; 18: 581-4.

43 Moene FC, Spinhoven P, Hoogduin KAL, Van Dyck R. A randomised controlled clinical trial on the additional effect of hypnosis in a comprehensive treatment programme for inpatients with conversion disorder of the motor type. Psychother Psychosom 2002; 71: 66-76.

44 Moene FC, Spinhoven P, Hoogduin KAL, Van Dyck R. A randomized controlled clinical trial of a hypnosis-based treatment for patients with conversion disorder, motor type. Int J Clin Exp Hypn 2003; 51: 29-50

45 Bleichhardt G, Timmer B, Rief W. Cognitive-behavioural therapy for patients with multiple somatoform symptoms. A randomized controlled trial in tertiary care. J Psychosom Res 2004; 56: 449-54.

46 Nickel M, Cangoez B, Bachler E, Muehlbacher M, Lojewski N, Mueller-Rabe N, et al. Bioenergetic exercises in inpatient treatment of Turkish immigrants with chronic somatoform disorders: a randomized, controlled study. J Psychosom Res 2006; 61: 507-13.

47 Zaby A, Heider J, Schroder A. Warten, Entspannung oder Verhaltenstherapie. Wie effektiv sind ambulante Gruppenbehandlungen bei multiplen somatoformen Symptomen [Waiting, relaxation, or behavioural therapy. How effective is out-patient group therapy for patients' somatoform symptoms]? Z KI Psych Psychoth 2008; 37: 15-23.

48 Luyten $\mathrm{P}$, Van Houdenhove $\mathrm{B}$, Lemma A, Target M, Fonagy P. A mentalizationbased approach to the understanding and treatment of functional somatic disorders. Psychoanal Psychother 2012; 26: 121-40.

49 Luyten $\mathrm{P}$, Van Houdenhove B. Common and specific factors in the psychotherapeutic treatment of patients suffering from chronic fatigue and pain. J Psychother Integrat 2013; 23: 14-27.

50 Hilsenroth MJ, Ackerman SJ, Blagys MD. Evaluating the phase model of change during short-term psychodynamic psychotherapy. Psychother Res 2001; 11: 29-47.

51 Perry JC, Bond M. The sequence of recovery in long-term dynamic psychotherapy. J Nerv Ment Dis 2009; 197: 930-7.

52 Driessen E, Cuijpers P, Maat SCM, Abbass AA, De Jonghe F, Dekker JJM. The efficacy of short-term psychodynamic psychotherapy for depression: a meta-analysis. Clin Psychol Rev 2010; 30: 25-36.

53 Subic-Wrana C, Bruder S, Thomas W, Lane RD, Kohle K. Emotional awareness deficits in inpatients of a psychosomatic ward: a comparison of two different measures of alexithymia. Psychosom Med 2005; 67: 483-9.

54 Lumley MA. Beyond cognitive-behavioral therapy for fibromyalgia: addressing stress by emotional exposure, processing, and resolution. Arthritis Res Ther 2011; 13: 136.

55 Rimes KA, Chalder T. The Beliefs about Emotions Scale: validity, reliability and sensitivity to change. J Psychosom Res 2010; 68: 285-92.

56 Noyes R, Langbehn DR, Happel RL, Stout LR, Muller BA, Longley SL. Personality dysfunction among somatizing patients. Psychosomatics 2001; 42: $320-9$.

57 Waller E, Scheidt CE, Hartmann A. Attachment representation and illness behavior in somatoform disorders. J Nerv Ment Dis 2004; 192: 200-9.

58 Blagys MD, Hilsenroth MJ. Distinctive activities of short-term psychodynamic interpersonal psychotherapy: a review of the comparative psychotherapy process literature. Clin Psychol Sci Pr 2000; 7: 167-88.

59 Diener MJ, Hilsenroth MJ, Weinberger J. Therapist affect focus and patient outcomes in psychodynamic psychotherapy: a meta-analysis. Am J Psychiatry 2007; 164: 936-41.

60 Dekker JJM, Koelen JA, Peen J, Schoevers RA, Gijsbers-Van Wijk C. Gender differences in clinical features of depressed outpatients: preliminary evidence for subtyping of depression? Women Health 2007; 46: 19-38.

61 Creed F, Guthrie E, Fink P, Henningsen P, Rief W, Sharpe $M$, et al. Is there a better term than 'medically unexplained symptoms'? J Psychosom Res 2010; 68: $5-8$.

62 Fink $P$, Schroder A. One single diagnosis, bodily distress syndrome, succeeded to capture 10 diagnostic categories of functional somatic syndromes and somatoform disorders. J Psychosom Res 2010; 68: 415-26. 